\title{
Improved Analytical Model of a Permanent-Magnet Brushless DC Motor
}

\author{
P. Kumar and P. Bauer \\ Delft University of Technology, 2628 CD Delft, The Netherlands
}

\begin{abstract}
In this paper, we develop a comprehensive model of a permanent-magnet brushless DC (BLDC) motor. An analytical model for determining instantaneous air-gap field density is developed. This instantaneous field distribution can be further used to determine the cogging torque, induced back electromotive force, and iron losses in the motor. The advantage of analytical models is that they can be readily used for optimization of BLDC motor because they are fast.
\end{abstract}

Index Terms-Air-gap field, BLDC motor, magnetization, permanent magnets.

\section{INTRODUCTION}

A general analytical technique for predicting the open circuit magnetic field distribution in radial field topologies of permanent-magnet brushless DC (BLDC) motor is a prerequisite for determination of force, and hence prediction of noises and vibration [1]. The instantaneous air-gap field density distribution is also required for calculation of induced back electromotive force (back-EMF) to determine the steady state and transient performance simulations [2]. Prediction of torque speed characteristics [3] as well as calculation of cogging torque magnitude and waveform [4] requires determination of instantaneous air-gap field distribution.

Eid and Mouilett [5] proposed an analytical model for predicting the two-dimensional air-gap field distribution of the internal rotor motors having a cylindrical permanent magnet with uniform diametric magnetization. In addition, Gu and Gao [6] used the method of separation of variables to analyze the air-gap field of a multipole permanent-magnet motor. His analysis was based on rectangular coordinates. Boules [7] also presented a model of multipole permanent-magnet motor in rectangular coordinates but used an equivalent magnet pole arc to take into account that in a cylindrical machine the circumferential width of the magnet generally varies with the radius. A further enhanced model was proposed by Boules [8]. In this model, he formulated a model in polar coordinates which utilizes the concept of equivalent current carrying coils to determine the flux density at the stator and rotor surfaces of a permanent-magnet motor. Zhu et al. [9] developed an improved analytical method for determination of field distribution. The methodology proposed by Zhu et al. was based on two-dimensional polar coordinates and addressed both internal and external rotor motor topologies. This technique involved solution of governing Laplacian/quasi-Poissonian field equations in the air-gap/magnet regions without any assumption regarding the relative recoil permeability of the magnets. However, the model of Zhu et al. [9] was only applicable to machines having radially magnetized magnets. Rasmussan [10] extended the model to account for the parallel magnetization. Recently, Zhu et al. [11] extended the model further to taker into account both radial and parallel magnetization.

Digital Object Identifier 10.1109/TMAG.2008.2001450

Color versions of one or more of the figures in this paper are available online at http://ieeexplore.ieee.org.
In the above discussed works of different authors, it was assumed that the permeability of iron was infinite and the thickness of stator iron is infinite. In the present work, we develop an analytical model for the instantaneous air-gap field density with the assumption that the iron (both stator and rotor yoke) has finite permeability and the thickness of the stator yoke is finite. Apart from that the presented model is valid for radial magnetization, parallel magnetization, radial sinusoidal amplitude magnetization, and sinusoidal angle magnetization. The results obtained by this model are closer to the reality and as shown in this work the results match with the results obtained from the finite-element method (FEM). This makes them effective for optimization process. The magnetic field distribution obtained from this model can be further utilized to determine the cogging torque, induced back-EMF, and iron losses. In the next section, magnetization of the permanent magnets are presented. The analytical model of the field distribution for a slotless stator is presented in Section III. Section IV deals with the development of the analytical model for a stator with slots, and the results of comparison between the analytical model and FEM results is also presented in this section. In Section V, the model to determine back-EMF using the results obtained in Section IV is given. The model of cogging torque and the comparison between analytical results and FEM is given in Section VI. Finally, conclusions are drawn in Section VII.

\section{Magnetization of Permanent Magnets}

The general configuration of a permanent-magnet brushless DC motor (BLDC) considered in the present work is shown in Fig. 1.

In the above figure, $\mu_{1}, \mu_{2}$, and $\mu_{3}$ represent the relative permeability of stator iron, permanent magnets, and rotor iron, respectively. The radii $R_{o}, R_{s}, R_{m}$, and $R_{r}$ represent outer radius of the motor, inner radius of the stator, radius of the magnets, and radius of the rotor, respectively. In the present analysis, it is assumed that the region exterior to the motor is air.

For the motor shown in Fig. 1, the magnetic field vector $H$ and magnetic field density vector $\boldsymbol{B}$ are coupled by the following set of equations:

$$
\begin{aligned}
& \boldsymbol{B}_{\boldsymbol{o}}=\mu_{0} \boldsymbol{H}_{\boldsymbol{o}} \text { in the exterior region } \\
& \boldsymbol{B}_{\boldsymbol{s}}=\mu_{0} \mu_{1} \boldsymbol{H}_{\boldsymbol{s}} \text { in the stator region } \\
& \boldsymbol{B}_{\boldsymbol{A}}=\mu_{0} \boldsymbol{H}_{\boldsymbol{A}} \text { in the air-gap region } \\
& \boldsymbol{B}_{\boldsymbol{M}}=\mu_{0} \mu_{2} \boldsymbol{H}_{\boldsymbol{M}}+\mu_{0} \boldsymbol{M} \text { in the magnet region } \\
& \boldsymbol{B}_{\boldsymbol{R}}=\mu_{0} \mu_{2} \boldsymbol{H}_{\boldsymbol{R}} \quad \text { in the rotor region }
\end{aligned}
$$




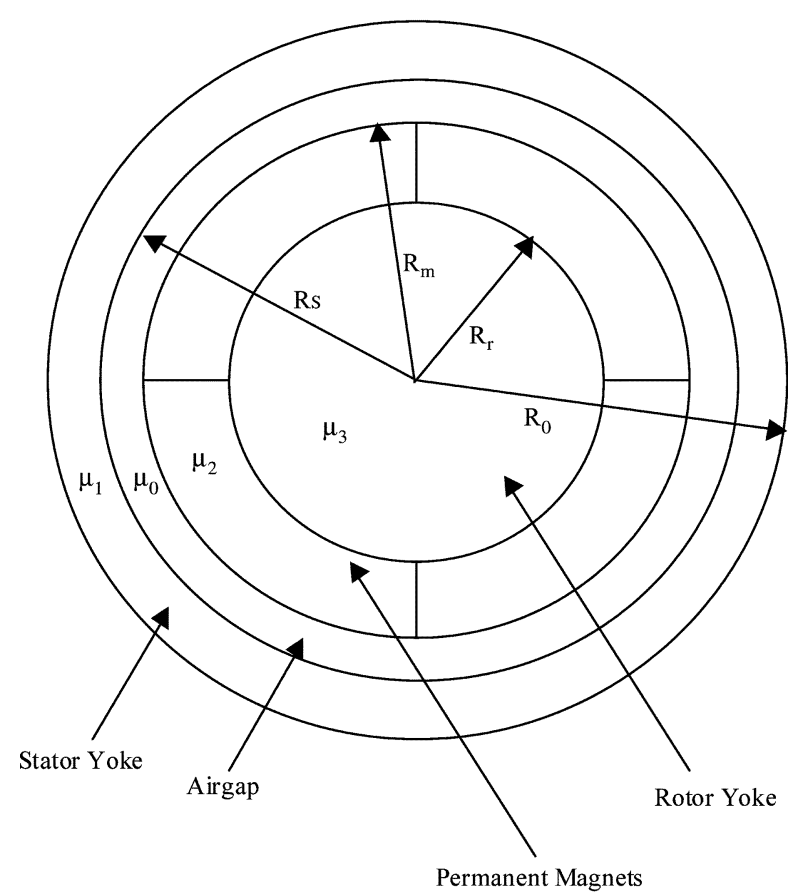

Fig. 1. General schematic diagram of a BLDC motor without slots.

where $\boldsymbol{M}$ is the magnetization vector of the permanent magnets. The amplitude of the magnetization vector $\boldsymbol{M}$, for a multipole motor with permanent magnets having a linear second quadrant demagnetization characteristics, is given by

$$
M=\frac{B_{r}}{\mu_{0}} .
$$

The direction of $\boldsymbol{M}$ depends on the orientation and magnetization of the permanent magnets. In polar coordinates, the magnetization vector $\boldsymbol{M}$ is expressed as

$$
M=M_{r} r+M_{\theta} \boldsymbol{\theta} .
$$

The different types of magnetization viz. radial magnetization, parallel magnetization, radial sinusoidal amplitude magnetization, and sinusoidal angle are discussed in [12].

\section{Field Produced IN A SLOtLesS Motor by MagnetS}

To facilitate the analysis and to obtain a closed form solution for the air-gap field distribution produced by magnets mounted on the rotor surface, the following assumptions are made.

1) The permanent magnets have a linear demagnetization characteristic.

2) End effects are neglected.

3) The stator and rotor back iron have constant permeability and the saturation is neglected.

The radial and tangential components of the magnetic field can be written in terms of scalar potential as follows:

$$
H_{r}=-\frac{\partial \varphi}{\partial r} \quad \text { and } \quad H_{\theta}=-\frac{1}{r} \frac{\partial \varphi}{\partial \theta} .
$$

The scalar magnetic potential distribution in the air gap, stator iron, rotor iron, and the exterior region is governed by the Laplace equation. In the magnet region, the scalar magnetic potential distribution is governed by quasi-Poissonian equation.

For the air-gap region, the Laplace equation is

$$
-\frac{\partial^{2} \varphi_{A}}{\partial r^{2}}+\frac{1}{r} \frac{\partial \varphi_{A}}{\partial r}+\frac{1}{r^{2}} \frac{\partial^{2} \varphi_{A}}{\partial \theta^{2}}=0 .
$$

In the magnet region, the quasi-Poissonian equation is

$$
-\frac{\partial^{2} \varphi_{M}}{\partial r^{2}}+\frac{1}{r} \frac{\partial \varphi_{M}}{\partial r}+\frac{1}{r^{2}} \frac{\partial^{2} \varphi_{M}}{\partial \theta^{2}}=\frac{1}{\mu_{2}} \nabla \cdot \boldsymbol{M} .
$$

In the stator iron region, the Laplace equation is

$$
-\frac{\partial^{2} \varphi_{S}}{\partial r^{2}}+\frac{1}{r} \frac{\partial \varphi_{S}}{\partial r}+\frac{1}{r^{2}} \frac{\partial^{2} \varphi_{S}}{\partial \theta^{2}}=0 .
$$

For the rotor iron region, the Laplace equation is

$$
-\frac{\partial^{2} \varphi_{R}}{\partial r^{2}}+\frac{1}{r} \frac{\partial \varphi_{R}}{\partial r}+\frac{1}{r^{2}} \frac{\partial^{2} \varphi_{R}}{\partial \theta^{2}}=0 .
$$

Finally for the exterior region, the Laplace equation is

$$
-\frac{\partial^{2} \varphi_{O}}{\partial r^{2}}+\frac{1}{r} \frac{\partial \varphi_{O}}{\partial r}+\frac{1}{r^{2}} \frac{\partial^{2} \varphi_{O}}{\partial \theta^{2}}=0
$$

where $\varphi_{A}, \varphi_{M}, \varphi_{s}, \varphi_{R}$, and $\varphi_{O}$ represent the magnetic scalar potential in the air gap, magnet, stator, rotor, and the exterior (outer) region, respectively.

From (2), we get

$$
\nabla \cdot \boldsymbol{M}=\frac{M_{r}}{r}+\frac{\partial M_{r}}{\partial r}+\frac{1}{r} \frac{\partial M_{\theta}}{\partial \theta}=\sum_{n=1,3,5, \ldots}^{\infty} \frac{1}{r} M_{n} \cos \left(n N_{p} \theta\right)
$$

where

$$
M_{n}=M_{r n}+n N_{p} M_{\theta n} .
$$

The boundary conditions for the motor shown in Fig. 1 are as follows:

(i) at the interface between the stator and the exterior region

$$
\begin{aligned}
\left.H_{\theta O}(r, \theta)\right|_{r=R_{o}} & =\left.H_{\theta S}(r, \theta)\right|_{r=R_{o}} \\
\left.B_{r O}(r, \theta)\right|_{r=R_{o}} & =\left.B_{r S}(r, \theta)\right|_{r=R_{o}}
\end{aligned}
$$

where $H_{\theta O}$ and $H_{\theta S}$ are the tangential component of the magnetic field vector in the exterior region and the stator iron, respectively, whereas $B_{r O}$ and $B_{r S}$ are the radial component of the magnetic field density vector in the exterior region and the stator iron, respectively.

(ii) at the interface between the stator and the air gap

$$
\begin{aligned}
\left.H_{\theta S}(r, \theta)\right|_{r=R_{S}} & =\left.H_{\theta A}(r, \theta)\right|_{r=R_{S}} \\
\left.B_{r S}(r, \theta)\right|_{r=R_{S}} & =\left.B_{r A}(r, \theta)\right|_{r=R_{S}}
\end{aligned}
$$

where $H_{\theta A}$ is the tangential component of the magnetic field vector in the air-gap region whereas $B_{r A}$ is the radial component of the magnetic field density vector in the air-gap region. 
(iii) at the interface between the air gap and the permanent magnet

$$
\begin{aligned}
\left.H_{\theta A}(r, \theta)\right|_{r=R_{M}} & =\left.H_{\theta M}(r, \theta)\right|_{r=R_{M}} \\
\left.B_{r A}(r, \theta)\right|_{r=R_{M}} & =\left.B_{r M}(r, \theta)\right|_{r=R_{M}}
\end{aligned}
$$

where $H_{\theta M}$ is the tangential component of the magnetic field vector in the magnet region whereas $B_{r M}$ is the radial component of the magnetic field density vector in the magnet region.

(iv) at the interface between the magnet and the rotor iron

$$
\begin{aligned}
\left.H_{\theta M}(r, \theta)\right|_{r=R_{r}} & =\left.H_{\theta R}(r, \theta)\right|_{r=R_{r}} \\
\left.B_{r M}(r, \theta)\right|_{r=R_{r}} & =\left.B_{r R}(r, \theta)\right|_{r=R_{r}}
\end{aligned}
$$

where $H_{\theta R}$ is the tangential component of the magnetic field vector in the rotor iron region whereas $B_{r R}$ is the radial component of the magnetic field density vector in the rotor iron region.

The dimensions $R_{r}, R_{S}, R_{M}$, and $R_{O}$ are depicted in Fig. 1 .

The general solution of the system of (5) and (6) are

$$
\begin{aligned}
\varphi_{O}(r, \theta) & =B_{n O} r^{-n N_{p}} \cos \left(n N_{p} \theta\right) \\
\varphi_{S}(r, \theta) & =\left(A_{n S} r^{n N_{p}}+B_{n S} r^{-n N_{p}}\right) \cos \left(n N_{p} \theta\right) \\
\varphi_{A}(r, \theta) & =\left(A_{n A} r^{n N_{p}}+B_{n A} r^{-n N_{p}}\right) \cos \left(n N_{p} \theta\right) \\
\varphi_{M}(r, \theta) & =\left(A_{n M} r^{n N_{p}}+B_{n M} r^{-n N_{p}}\right) \cos \left(n N_{p}\right) \\
& +\frac{M_{n} r \cos \left(n N_{p} \theta\right)}{\mu_{2}\left(1-n^{2} N_{p}^{2}\right)} \\
\varphi_{R}(r, \theta) & =A_{n R} r^{n N_{p}} \cos \left(n N_{p} \theta\right)
\end{aligned}
$$

where $B_{n O}, A_{n S}, B_{n S}, A_{n A}, B_{n A}, A_{n M}, B_{n M}$, and $A_{n R}$ are constants to be determined. These constants are determined by solving the boundary conditions given in (8) and using (2) and (1). Upon substituting back the constants $A_{n A}$ and $B_{n A}$ into (11) and using (4) and (1), the air radial and tangential components of the air-gap field distribution are obtained as follows:

$$
\begin{aligned}
& B_{r}(r, \theta)=\sum_{n=1,3,5, \ldots}^{\infty}\left(n N_{p}\right) \mu_{0} M_{n} \frac{B_{1} B_{2}}{B_{3}} \cos \left(n N_{p} \theta\right) \\
& B_{\theta}(r, \theta)=\sum_{n=1,3,5, \ldots}^{\infty}\left(n N_{p}\right) \mu_{0} M_{n} \frac{B_{1} B_{4}}{B_{3}} \sin \left(n N_{p} \theta\right)
\end{aligned}
$$

where

$$
\begin{aligned}
B_{1}=R_{m} R_{r}^{\left(n N_{p}+1\right)}[2 & \left(\frac{R_{m}}{R_{r}}\right)^{n N_{p}-1}-\left(n N_{p}+1\right) \\
& \left.+\left(\frac{R_{m}}{R_{r}}\right)^{2 n N_{p}}\left(n N_{p}-1\right)\right] \mu_{3} \\
-R_{m} R_{r}^{\left(n N_{p}+1\right)} & {\left[n N_{p}-\left(\frac{R_{r}}{R_{m}}\right)^{n N_{p}-1}\left(n N_{p}+1\right)\right.} \\
& \left.-\left(\frac{R_{m}}{R_{r}}\right)^{n N_{p}+1}\left(n N_{p}-1\right)\right] \mu_{1}
\end{aligned}
$$

$$
\begin{aligned}
& B_{2}=\left(\frac{r}{R_{s}}\right)^{n N_{p}}\left(\frac{R_{o}}{R_{s}}\right)^{n N_{p}}\left(\mu_{2}^{2}-1\right) \\
& +\left[\left(1-\mu_{2}^{2}\right)\left(\frac{r}{R_{o}}\right)^{n N_{p}}+\left(1+\mu_{2}\right)^{2}\left(\frac{R_{o}}{r}\right)^{n N_{p}}\right] \\
& -\left(\frac{R_{s}}{r}\right)^{n N_{p}}\left(\frac{R_{s}}{R_{o}}\right)^{n N_{p}}\left(1-\mu_{2}\right)^{2} \\
& B_{3}=r\left[\left(K_{1}+K_{2}\right) K_{3}+\left(K_{4}+K_{5}\right) K_{6}\right]\left(1-n^{2} N_{p}^{2}\right) \\
& K_{1}=\left(1+\mu_{1}\right)\left(\frac{R_{o}}{R_{s}}\right)^{n N_{p}} \\
& \times\left[\left(1+\mu_{2}\right)^{2}-\left(1-\mu_{2}\right)^{2}\left(\frac{R_{s}}{R_{o}}\right)^{2 n N_{p}}\right] \\
& K_{2}=\left(\mu_{1}-1\right)\left(\mu_{2}^{2}-1\right)\left(\frac{R_{m}}{R_{r}}\right)^{n N_{p}}\left(\frac{R_{m}}{R_{s}}\right)^{n N_{p}} \\
& \times\left[\left(\frac{R_{o}}{R_{S}}\right)^{n N_{p}}-\left(\frac{R_{s}}{R_{o}}\right)^{n N_{p}}\right] \\
& K_{3}=\mu_{3} R_{r} R_{m}^{n N_{p}}+\mu_{1} R_{m} R_{r}^{n N_{p}} \\
& K_{4}=\left(1+\mu_{1}\right)\left(\frac{R_{r}}{R_{m}}\right)^{n N_{p}}\left(\frac{R_{o}}{R_{m}}\right)^{n N_{p}} \\
& \times\left[\left(1+\mu_{2}\right)^{2}-\left(1-\mu_{2}\right)^{2}\left(\frac{R_{s}}{R_{o}}\right)^{2 n N_{p}}\right] \\
& K_{5}=\left(\mu_{1}+1\right)\left(\mu_{2}^{2}-1\right)\left(\frac{R_{r}}{R_{s}}\right)^{n N_{p}} \\
& \times\left[\left(\frac{R_{o}}{R_{S}}\right)^{n N_{p}}-\left(\frac{R_{s}}{R_{o}}\right)^{n N_{p}}\right] \\
& K_{6}=\mu_{1} R_{m} R_{r}^{n N_{p}}-\mu_{3} R_{r} R_{m}^{n N_{p}} \\
& B_{4}=\left(\frac{r}{R_{s}}\right)^{n N_{p}}\left(\frac{R_{o}}{R_{s}}\right)^{n N_{p}}\left(\mu_{2}^{2}-1\right) \\
& +\left[\left(1-\mu_{2}^{2}\right)\left(\frac{r}{R_{o}}\right)^{n N_{p}}-\left(1+\mu_{2}\right)^{2}\left(\frac{R_{o}}{r}\right)^{n N_{p}}\right] \\
& +\left(\frac{R_{s}}{r}\right)^{n N_{p}}\left(\frac{R_{s}}{R_{o}}\right)^{n N_{p}}\left(1-\mu_{2}\right)^{2}
\end{aligned}
$$

when $n N_{p}=1$ the field distribution in the air gap is given by

$$
\begin{aligned}
& B_{r}(r, \theta)=\frac{1}{2} \mu_{o} M_{n}\left(\frac{R_{r}}{r}\right)^{2} \frac{B_{1} B_{2}}{B_{3}} \cos (\theta) \\
& B_{\theta}(r, \theta)=\frac{1}{2} \mu_{o} M_{n}\left(\frac{R_{r}}{r}\right)^{2} \frac{B_{1} B_{4}}{B_{3}} \cos (\theta)
\end{aligned}
$$

where

$$
\begin{aligned}
B_{1}= & 2\left(\mu_{3}-\mu_{1}\right) \ln \left(\frac{R_{m}}{R_{r}}\right)+\left(\frac{R_{m}^{2}}{R_{r}^{2}}-1\right)\left(\mu_{1}+\mu_{3}\right) \\
B_{2}= & \left(\mu_{2}-1\right)^{2}+\left(\frac{r^{2}}{R_{s}^{2}}-\frac{R_{o}^{2}}{R_{s}^{2}}-\frac{r^{2}}{R_{s}^{2}} \frac{R_{o}^{2}}{R_{s}^{2}}\right) \mu_{2}^{2} \\
& -2\left(\frac{R_{o}}{R_{s}}\right)^{2} \mu_{2}-\frac{r^{2}}{R_{s}^{2}}-\frac{R_{o}^{2}}{R_{s}^{2}}+\frac{r^{2}}{R_{s}^{2}} \frac{R_{o}^{2}}{R_{s}^{2}}
\end{aligned}
$$




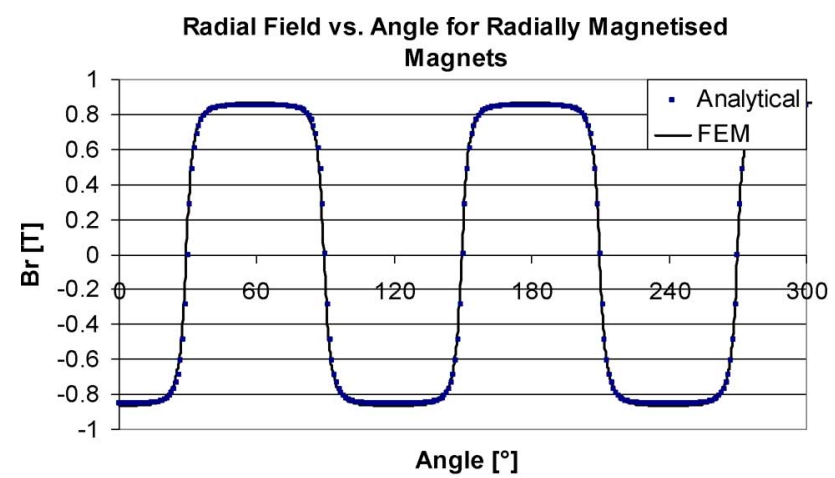

(a)

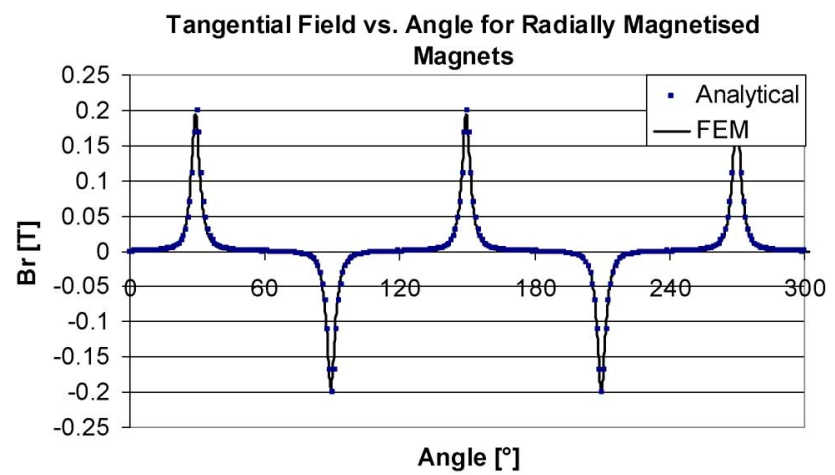

(b)

Fig. 2. Distribution of (a) radial component and (b) tangential component of air-gap field in a slotless motor with radially magnetized magnets in air gap $(\mathrm{r}=16.75 \mathrm{~mm})$. .

$$
\begin{aligned}
B_{3}= & \left(\mu_{2}-1\right)^{2}\left(\mu_{1}^{2}\left(\frac{R_{m}^{2}}{R_{r}^{2}}-1\right)+\left(\mu_{1}+\mu_{3}\right) \mu_{1}\left(\frac{R_{m}^{2}}{R_{r}^{2}}+1\right)\right. \\
& \left.+\left(\frac{R_{m}^{2}}{R_{r}^{2}}-1\right) \mu_{3}\right) \\
B_{2}= & \left(\mu_{2}-1\right)^{2}+\left(-\frac{r^{2}}{R_{s}^{2}}-\frac{R_{o}^{2}}{R_{s}^{2}}-\frac{r^{2}}{R_{s}^{2}} \frac{R_{o}^{2}}{R_{s}^{2}}\right) \mu_{2}^{2} \\
& -2\left(\frac{R_{o}}{R_{s}}\right)^{2} \mu_{2}+\frac{r^{2}}{R_{s}^{2}}-\frac{R_{o}^{2}}{R_{s}^{2}}-\frac{r^{2}}{R_{s}^{2}} \frac{R_{o}^{2}}{R_{s}^{2}} .
\end{aligned}
$$

This model has been applied to three-phase slotless BLDC motor with radial, parallel, sinusoidal magnitude, and sinusoidal angle magnetization. The parameters of the motor taken are

$$
\begin{aligned}
& N_{p}=6, \quad R_{s}=x, \quad R_{r}=y, \quad R_{m}=z, \quad B_{r}=1.2 \mathrm{~T}, \\
& \mu_{1}=1000, \quad \mu_{2}=1.05, \quad \mu_{3}=1000, \alpha_{m}=0.8 .
\end{aligned}
$$

The results obtained by our model and the FEM are shown in Fig. 2 for radial magnetization, Fig. 3 for parallel magnetization, Fig. 4 for sinusoidal angle magnetization, and Fig. 5 for sinusoidal amplitude magnetization.

\section{Field Produced in a Slotted Motor by Magnets}

In the case of a slotted stator (Fig. 6) the magnetic field is changed throughout the air gap and magnet region due to presence of slots. The change in the magnetic field due to slotting

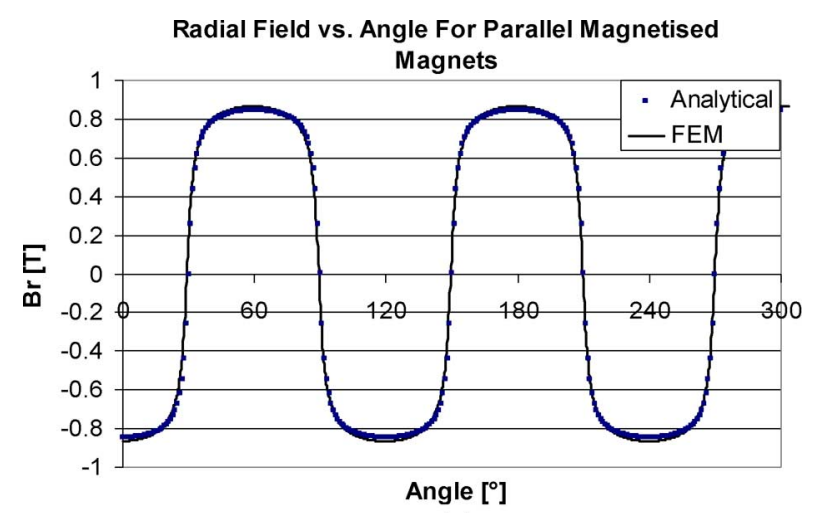

(a)

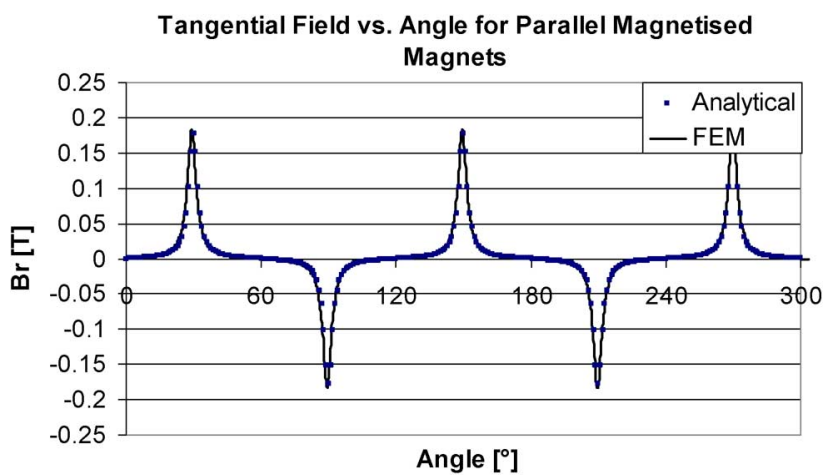

(b)

Fig. 3. Distribution of (a) radial component and (b) tangential component of air-gap field in a slotless motor with parallel magnetized magnet $(\mathrm{r}=16.75 \mathrm{~mm})$.

is a function of distance from the slots. The influence of slots is minimum at the magnet and rotor iron interface, whereas the greatest influence of slots is experienced at the stator surface. Besides this the slotting is a function of saturation of the ferromagnetic material used in the rotor and stator. Since saturation effect is very difficult to describe analytically, it has been ignored in the present analysis.

The fundamental principle governing the influence of the stator slots is the fact that the magnetic field over the slots must travel further to reach the stator ferromagnetic material. In other words, the air gap is longer over the slots. As a result of this larger effective air gap, the flux density is reduced over the slot area. Zhu et al. [13] have discussed in great detail the slotting effect for a permanent-magnet motor. To accommodate the effects of slotting they developed a correction factor known as relative permeance.

Lieu et al. [14] developed an analytical model of tangential air-gap field due to slotting. However, in their model they assumed that the stator iron to be of infinite thickness and its permeability to be infinite. In the present work, as stated before, the stator iron is assumed to have finite thickness and finite permeability. The analysis starts with the calculation of the radial flux density along the outer surface of a smooth stator as discussed in the previous section. The actual radial flux density at the stator surface is calculated by multiplying the relative permeance function with the radial flux density of a slotless motor 


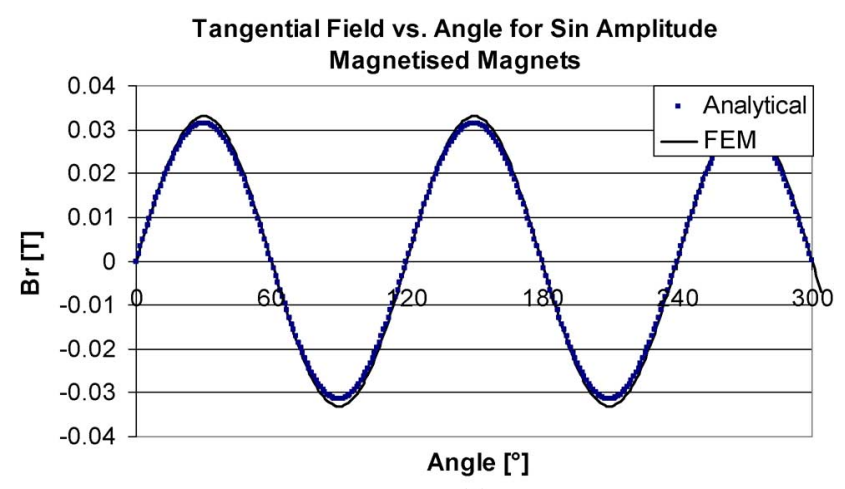

(a)

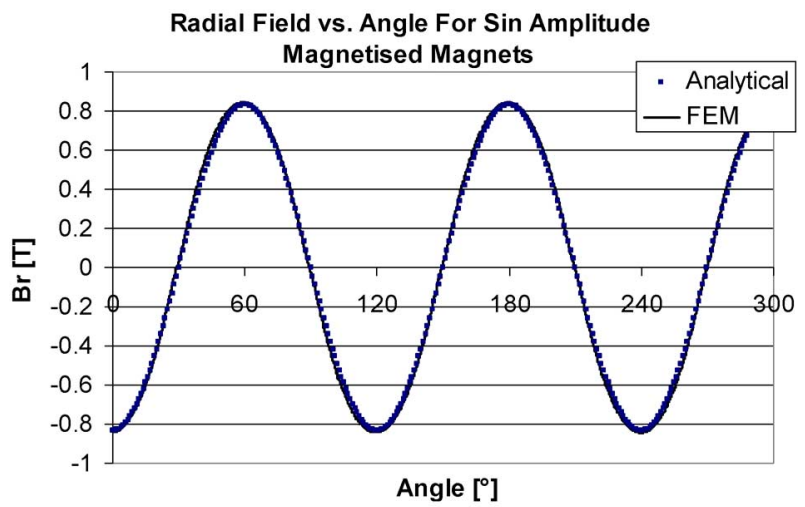

(b)

Fig. 4. Distribution of (a) radial component and (b) tangential component of air-gap field in slotless motor with sin amplitude magnetized magnets $(\mathbf{r}=$ $16.75 \mathrm{~mm}$ ).

$\left(B_{r}(r, \theta)\right.$ in the previous section). This actual radial flux density is considered as a new boundary condition and hence a new boundary value problem is set up

$$
B_{r I}(r, \theta)=B_{r}(r, \theta) \lambda_{m}(r, \theta)
$$

where $B_{r}(r, \theta)$ is given by (14) in case $n N_{p} \neq 1$ and in case $n N_{p}=1$ by (16) and $\lambda_{m}(r, \theta)$ is the relative permeance function as defined by Zhu et al. [13]. At the stator surface

$$
\begin{aligned}
\left.B_{r I}(r, \theta)\right|_{r=R_{s}}= & \left\{\sum_{n=1,3,5, \ldots}^{\infty} B_{n} \cos \left(n N_{p} \theta\right)\right\} \\
& \times\left\{\sum_{m=0,1,2, \ldots}^{\infty} \lambda_{m} \cos \left(m N_{s} \theta\right)\right\}
\end{aligned}
$$

where $N_{s}$ is the number of slots on the stator.

The above equation can also be written as

$$
\begin{aligned}
& \left.B_{r I}(r, \theta)\right|_{r=R_{s}} \\
& =\sum_{n=1,3,5, \ldots}^{\infty} B_{n} \lambda_{o} \cos \left(n N_{p} \theta\right) \\
& \quad+\frac{1}{2} \sum_{n=1,3,5, \ldots}^{\infty} \sum_{m=1,2,3, \ldots}^{\infty} B_{n} \lambda_{m} \\
& \quad \cdot\left\{\cos \left[\left(n N_{p}+m N_{s}\right) \theta\right]+\cos \left[\left(n N_{p}-m N_{s}\right) \theta\right]\right\}
\end{aligned}
$$

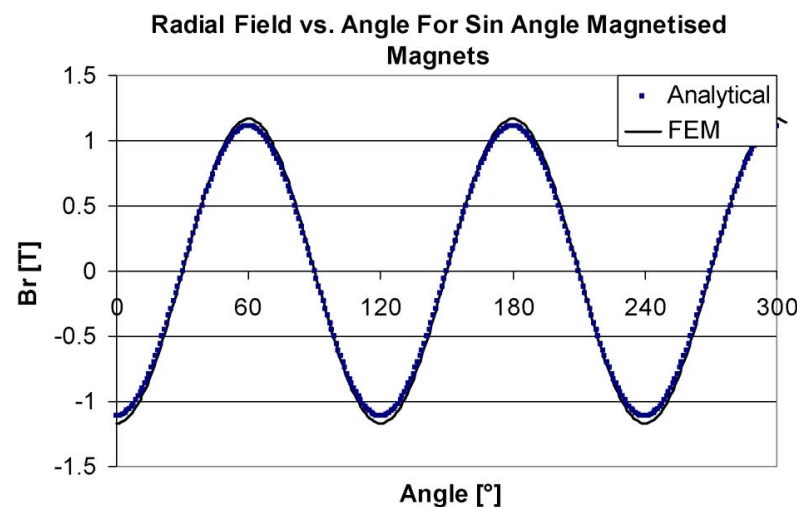

(a)

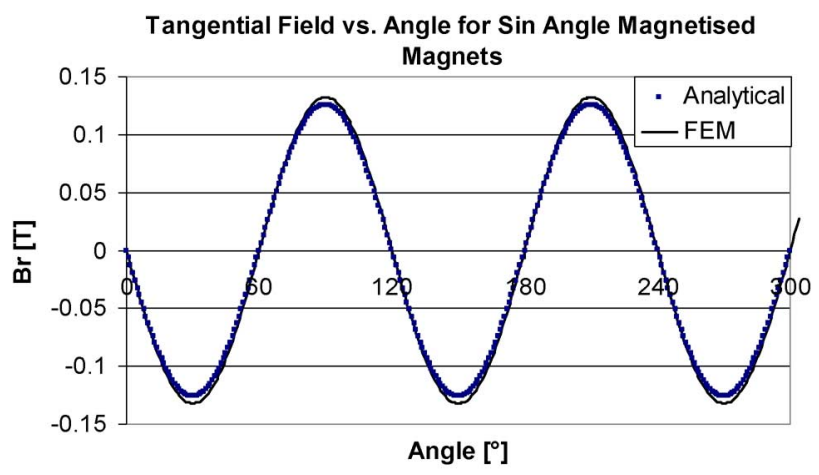

(b)

Fig. 5. Distribution of (a) radial component and (b) tangential component of air-gap field in a slotless motor with parallel magnetized magnets in air gap $(\mathrm{r}=16.75 \mathrm{~mm})$.

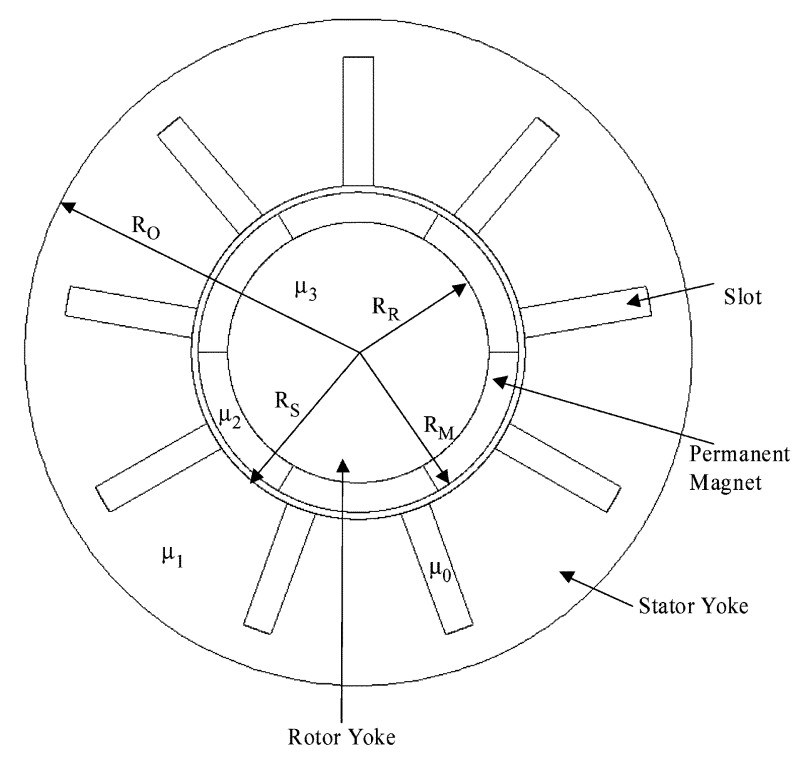

Fig. 6. General schematic diagram of a BLDC motor with slots.

where $B_{n}$ is the magnitude flux density at the stator surface and can be obtained by substituting $r$ by $R_{s}$ in (14) in case $n N_{p} \neq 1$ and in case $n N_{p}=1$ by $(16) ; \lambda_{m}$ is the amplitude of the relative permeance function as given by Zhu et al. [13]. The scalar magnetic potential distribution in the air gap, stator iron, rotor iron, and the exterior region is governed by the Laplace equation. In the magnet region, the scalar magnetic potential 
distribution is governed by quasi-Poissonian equation. These set of equations have already been discussed in the previous section (5), but for the sake of continuity they are reproduced below.

For the air-gap region, the Laplace equation is

$$
-\frac{\partial^{2} \varphi_{A}}{\partial r^{2}}+\frac{1}{r} \frac{\partial \varphi_{A}}{\partial r}+\frac{1}{r^{2}} \frac{\partial^{2} \varphi_{A}}{\partial \theta^{2}}=0 .
$$

In the magnet region, the quasi-Poissonian equation is

$$
-\frac{\partial^{2} \varphi_{M}}{\partial r^{2}}+\frac{1}{r} \frac{\partial \varphi_{M}}{\partial r}+\frac{1}{r^{2}} \frac{\partial^{2} \varphi_{M}}{\partial \theta^{2}}=\frac{1}{\mu_{2}} \nabla \cdot \boldsymbol{M} .
$$

In the stator iron region, the Laplace equation is

$$
-\frac{\partial^{2} \varphi_{S}}{\partial r^{2}}+\frac{1}{r} \frac{\partial \varphi_{S}}{\partial r}+\frac{1}{r^{2}} \frac{\partial^{2} \varphi_{S}}{\partial \theta^{2}}=0 .
$$

For the rotor iron region, the Laplace equation is

$$
-\frac{\partial^{2} \varphi_{R}}{\partial r^{2}}+\frac{1}{r} \frac{\partial \varphi_{R}}{\partial r}+\frac{1}{r^{2}} \frac{\partial^{2} \varphi_{R}}{\partial \theta^{2}}=0 .
$$

Finally, for the exterior region the Laplace equation is

$$
-\frac{\partial^{2} \varphi_{O}}{\partial r^{2}}+\frac{1}{r} \frac{\partial \varphi_{O}}{\partial r}+\frac{1}{r^{2}} \frac{\partial^{2} \varphi_{O}}{\partial \theta^{2}}=0 .
$$

The general solution of the above equations is similar to that given in the previous section except that certain modifications are made to accommodate the slotting effect

$$
\begin{aligned}
\varphi_{O}(r, \theta) & =\varphi_{O 1}(r, \theta)+\varphi_{O 2}(r, \theta)+\varphi_{O 3}(r, \theta) \\
\varphi_{S}(r, \theta) & =\varphi_{S 1}(r, \theta)+\varphi_{S 2}(r, \theta)+\varphi_{S 3}(r, \theta) \\
\varphi_{A}(r, \theta) & =\varphi_{A 1}(r, \theta)+\varphi_{A 2}(r, \theta)+\varphi_{A 3}(r, \theta) \\
\varphi_{M}(r, \theta) & =\varphi_{M 1}(r, \theta)+\varphi_{M 2}(r, \theta)+\varphi_{M 3}(r, \theta) \\
\varphi_{R}(r, \theta) & =\varphi_{R 1}(r, \theta)+\varphi_{R 2}(r, \theta)+\varphi_{R 3}(r, \theta)
\end{aligned}
$$

where

$$
\begin{aligned}
\varphi_{O 1}(r, \theta)= & B_{n O 1} r^{-n N_{p}} \cos \left(n N_{p} \theta\right) \\
\varphi_{O 2}(r, \theta)= & B_{n O 2} r^{-n N_{p}-m N_{s}} \cos \left(n N_{p} \theta+m N_{s} \theta\right) \\
\varphi_{O 3}(r, \theta)= & B_{n O 3} r^{-n N_{p}+m N_{s}} \cos \left(n N_{p} \theta-m N_{s} \theta\right) \\
\varphi_{S 1}(r, \theta)= & \left(A_{n S 1} r^{n N_{p}}+B_{n S 1} r^{-n N_{p}}\right) \cos \left(n N_{p} \theta\right) \\
\varphi_{S 2}(r, \theta)= & \left(A_{n S 2} r^{n N_{p}+m N_{S}}+B_{n S 2} r^{-n N_{p}-m N_{S}}\right) \\
& \times \cos \left(n N_{p} \theta+m N_{S} \theta\right) \\
\varphi_{S 3}(r, \theta)= & \left(A_{n S 3} r^{n N_{p}-m N_{S}}+B_{n S 3} r^{-n N_{p}+m N_{S}}\right) \\
& \times \cos \left(n N_{p} \theta-m N_{S} \theta\right) \\
\varphi_{A 1}(r, \theta)= & \left(A_{n A 1} r^{n N_{p}}+B_{n A 1} r^{-n N_{p}}\right) \cos \left(n N_{p} \theta\right) \\
\varphi_{A 2}(r, \theta)= & \left(A_{n A 2} r^{n N_{p}+m N_{s}}+B_{n A 2} r^{-n N_{p}-m N_{s}}\right) \\
& \times \cos \left(n N_{p} \theta+m N_{s} \theta\right) \\
\varphi_{A 3}(r, \theta)= & \left(A_{n A 3} r^{n N_{p}-m N_{s}}+B_{n A 3} r^{-n N_{p}+m N_{s}}\right) \\
& \times \cos \left(n N_{p} \theta-m N_{s} \theta\right) \\
\varphi_{M 1}(r, \theta)= & \left(A_{n M 1} r^{n N_{p}}+B_{n M 1} r^{-n N_{p}}\right) \cos \left(n N_{p} \theta\right) \\
& +\frac{M_{n} r \cos \left(n N_{p} \theta\right)}{\mu_{2}\left(1-n^{2} N_{p}^{2}\right)}
\end{aligned}
$$

$$
\begin{aligned}
\varphi_{M 2}(r, \theta)= & \left(A_{n M 2} r^{n N_{p}+m N_{s}}+B_{n M 2} r^{-n N_{p}-m N_{s}}\right) \\
& \times \cos \left(n N_{p} \theta+m N_{s} \theta\right) \\
\varphi_{M 3}(r, \theta)= & \left(A_{n M 3} r^{n N_{p}-m N_{s}}+B_{n M 3} r^{-n N_{p}+m N_{s}}\right) \\
& \times \cos \left(n N_{p} \theta-m N_{s} \theta\right) \\
\varphi_{R 1}(r, \theta)= & A_{n R 1} r^{n N_{p}} \cos \left(n N_{p} \theta\right) \\
\varphi_{R 2}(r, \theta)= & A_{n R 2} r^{n N_{p}+m N_{s}} \cos \left(n N_{p} \theta+m N_{s} \theta\right) \\
\varphi_{R}(r, \theta)= & A_{n R 2} r^{n N_{p}-m N_{s}} \cos \left(n N_{p} \theta-m N_{s} \theta\right)
\end{aligned}
$$

where $B_{n O 1}, B_{n O 2}, B_{n O 3}, A_{n S 1}, B_{n S 1}, A_{n S 2}, B_{n s 2}, A_{n S 3}$, $B_{n S 3} A_{n A 1}, B_{n A 1}, A_{n A 2}, B_{n A 2}, A_{n A 3}, B_{n A 3}, A_{n M 1}, B_{n M 1}$, $A_{n M 2}, B_{n M 2} A_{n M 3}, B_{n M 3}, A_{n R 1}, A_{n R 2}$, and $A_{n R 3}$ are the constants to be determined.

The boundary conditions for this case are:

(i) at the interface between the stator and the exterior region

$$
\begin{gathered}
-\left.\frac{1}{r} \frac{\partial \varphi_{o i}}{\partial \theta}\right|_{r=R_{o}}=-\left.\frac{1}{r} \frac{\partial \varphi_{S i}}{\partial \theta}\right|_{r=R_{o}} i=1,2,3 \\
-\left.\mu_{o} \frac{\partial \varphi_{o 1}}{\partial r}\right|_{r=R_{o}}=-\left.\mu_{o} \mu_{1} \frac{\partial \varphi_{S 1}}{\partial r}\right|_{r=R_{o}} \quad i=1,2,3
\end{gathered}
$$

(ii) at the interface between the stator and the air gap

$$
\begin{aligned}
&-\left.\frac{1}{r} \frac{\partial \varphi_{S 1}}{\partial \theta}\right|_{r=R_{s}}=-\left.\frac{1}{r} \frac{\partial \varphi_{A 1}}{\partial \theta}\right|_{r=R_{s}} i=1,2,3 \\
&-\left.\mu_{o} \mu_{1} \frac{\partial \varphi_{S 1}}{\partial r}\right|_{r=R_{s}}= \sum_{n=1,3,5, \ldots}^{\infty} B_{n} \lambda_{o} \cos \left(n N_{p} \theta\right) \\
&-\left.\mu_{o} \mu_{1} \frac{\partial \varphi_{S 2}}{\partial r}\right|_{r=R_{s}}= \frac{1}{2} \sum_{n=1,3,5, \ldots}^{\infty} \sum_{m=1,2,3, \ldots}^{\infty} B_{n} \lambda_{m} \\
&-\left.\mu_{o} \mu_{1} \frac{\partial \varphi_{S 3}}{\partial r}\right|_{r=R_{s}}= \frac{1}{2} \sum_{n=1,3,5, \ldots m=1,2,3, \ldots}^{\infty} B_{n} \lambda_{m} \\
& \cdot \cos \left(n N_{p} \theta-n N_{s} \theta\right)
\end{aligned}
$$

(iii) at the interface between the air gap and the permanent magnet

$$
\begin{gathered}
-\left.\frac{1}{r} \frac{\partial \varphi_{A 1}}{\partial \theta}\right|_{r=R_{M}}=-\left.\frac{1}{r} \frac{\partial \varphi_{M 1}}{\partial \theta}\right|_{r=R_{M}} i=1,2,3 \\
-\left.\mu_{O} \frac{\partial \varphi_{A 1}}{\partial r}\right|_{r=R_{M}}=-\left.\mu_{o} \mu_{2} \frac{\partial \varphi_{M 1}}{\partial r}\right|_{r=R_{M}} i=1,2,3
\end{gathered}
$$

(iv) at the interface between the magnet and the rotor iron

$$
\begin{gathered}
-\left.\frac{1}{r} \frac{\partial \varphi_{M 1}}{\partial \theta}\right|_{r=R_{r}}=-\left.\frac{1}{r} \frac{\partial \varphi_{R 1}}{\partial \theta}\right|_{r=R_{r}} i=1,2,3 \\
-\left.\mu_{o} \mu_{2} \frac{\partial \varphi_{M 1}}{\partial r}\right|_{r=R_{r}}=-\left.\mu_{o} \mu_{3} \frac{\partial \varphi_{R 1}}{\partial r}\right|_{r=R_{r}} i=1,2,3 .
\end{gathered}
$$

The constants mentioned above are determined by solving the boundary conditions given in (42) to (55). Upon substituting back the constants $A_{n S 1}, B_{n S 1}, A_{n S 2}, B_{n s 2}, A_{n S 3}$, and $B_{n S 3}$ into (24) and using (4) and (1), the tangential components of the 
air-gap field distribution for the slotted case at the stator surface is obtained:

$$
\begin{aligned}
B_{\theta}(r, \theta) & =\sum_{n=1,3,5, \ldots}^{\infty} B_{I} \sin \left(n N_{p} \theta\right) \\
+\sum_{n=1,3,5, \ldots}^{\infty} \sum_{m=1,2,3, \ldots}^{\infty} B_{I I} & \begin{array}{r}
\sin \left(n N_{p} \theta+m N_{s} \theta\right) \\
\left.+\sin \left(n N_{p} \theta-m N_{s} \theta\right)\right\}
\end{array}
\end{aligned}
$$

where

$$
\begin{aligned}
& B_{I}=\left(n N_{p}\right) \mu_{0} M_{n} \frac{B_{1} B_{2}}{B_{3}} \\
& B_{1}=R_{m} R_{r}^{\left(n N_{p}+1\right)}\left[2\left(\frac{R_{m}}{R_{r}}\right)^{n N_{p}-1}-\left(n N_{p}+1\right)\right. \\
& \left.+\left(\frac{R_{m}}{R_{r}}\right)^{2 n N_{p}}\left(n N_{p}-1\right)\right] \mu_{3} \\
& -R_{m} R_{r}^{\left(n N_{p}+1\right)}\left[n N_{p}-\left(\frac{R_{r}}{R_{m}}\right)^{n N_{p}-1}\left(n N_{p}+1\right)\right. \\
& \left.-\left(\frac{R_{m}}{R_{r}}\right)^{n N_{p}+1}\left(n N_{p}-1\right)\right] \mu_{1} \\
& B_{2}=\left(\frac{R_{o}}{R_{s}}\right)^{n N_{p}}\left(\mu_{2}^{2}-1\right) \\
& +\left[\left(1-\mu_{2}^{2}\right)\left(\frac{R_{s}}{R_{o}}\right)^{n N_{p}}-\left(1+\mu_{2}\right)^{2}\left(\frac{R_{o}}{R_{s}}\right)^{n N_{p}}\right] \\
& +\left(\frac{R_{s}}{R_{o}}\right)^{n N_{p}}\left(1-\mu_{2}\right)^{2} \\
& B_{3}=r\left[\left(K_{1}+K_{2}\right) K_{3}+\left(K_{4}+K_{5}\right) K_{6}\right]\left(1-n^{2} N_{p}^{2}\right) \\
& K_{1}=\left(1+\mu_{1}\right)\left(\frac{R_{o}}{R_{s}}\right)^{n N_{p}} \\
& \times\left[\left(1+\mu_{2}\right)^{2}-\left(1-\mu_{2}\right)^{2}\left(\frac{R_{s}}{R_{o}}\right)^{2 n N_{p}}\right] \\
& K_{2}=\left(\mu_{1}-1\right)\left(\mu_{2}^{2}-1\right)\left(\frac{R_{m}}{R_{r}}\right)^{n N_{p}}\left(\frac{R_{m}}{R_{s}}\right)^{n N_{p}} \\
& \times\left[\left(\frac{R_{o}}{R_{S}}\right)^{n N_{p}}-\left(\frac{R_{s}}{R_{o}}\right)^{n N_{p}}\right] \\
& K_{3}=\mu_{3} R_{r} R_{m}^{n N_{p}}+\mu_{1} R_{m} R_{r}^{n N_{p}} \\
& K_{4}=\left(1+\mu_{1}\right)\left(\frac{R_{r}}{R_{m}}\right)^{n N_{p}}\left(\frac{R_{o}}{R_{m}}\right)^{n N_{p}} \\
& \times\left[\left(1+\mu_{2}\right)^{2}-\left(1-\mu_{2}\right)^{2}\left(\frac{R_{s}}{R_{o}}\right)^{2 n N_{p}}\right] \\
& K_{5}=\left(\mu_{1}+1\right)\left(\mu_{2}^{2}-1\right)\left(\frac{R_{r}}{R_{s}}\right)^{n N_{p}} \\
& \times\left[\left(\frac{R_{o}}{R_{S}}\right)^{n N_{p}}-\left(\frac{R_{s}}{R_{o}}\right)^{n N_{p}}\right] \\
& K_{6}=\mu_{1} R_{m} R_{r}^{n N_{p}}-\mu_{3} R_{r} R_{m}^{n N_{p}}
\end{aligned}
$$

$$
\begin{aligned}
B_{I I}= & B_{4} / B_{5} \\
B_{4}= & -\left(\frac{R_{R}}{R_{M}}\right)^{n N_{p}+m N_{s}}\left(\frac{R_{S}}{R_{M}}\right)^{n N_{p}+m N_{s}} \\
& \times\left(\mu_{2}-1\right)\left(\mu_{2}-\mu_{3}\right)+\left(\mu_{2}+1\right) \\
& \times\left(\left(\mu_{2}-\mu_{3}\right)\left(\frac{R_{R}}{R_{S}}\right)^{n N_{p}+m N_{s}}\right. \\
& \left.+\left(\mu_{2}+\mu_{3}\right)\left(\frac{R_{S}}{R_{R}}\right)^{n N_{p}+m N_{s}}\right) \\
- & \left(\frac{R_{M}}{R_{S}}\right)^{n N_{p}+m N_{s}}\left(\frac{R_{M}}{R_{R}}\right)^{n N_{p}+m N_{s}} \\
\times & \left(\mu_{2}-1\right)\left(\mu_{2}+\mu_{3}\right) \\
B_{5}= & \left(\mu_{2}-1\right)\left(\mu_{3}-\mu_{2}\right)\left(\frac{R_{s}}{R_{M}}\right)^{n N_{p}+m N_{s}}\left(\frac{R_{R}}{R_{M}}\right)^{n N_{p}+m N_{s}} \\
+ & \left(\mu_{2}-1\right)\left(\mu_{3}+\mu_{2}\right)\left(\frac{R_{M}}{R_{S}}\right)^{n N_{p}+m N_{s}}\left(\frac{R_{M}}{R_{R}}\right)^{n N_{p}+m N_{s}} \\
- & \left(\mu_{2}+1\right)\left(\mu_{3}+\mu_{2}\right)\left(\frac{R_{s}}{R_{R}}\right)^{n N_{p}+m N_{s}} \\
- & \left(\mu_{2}+1\right)\left(\mu_{3}-\mu_{2}\right)\left(\frac{R_{R}}{R_{S}}\right)^{n N_{p}+m N_{s}} \cdot
\end{aligned}
$$

The above developed model was applied to a three-phase slotless BLDC motor with radial, parallel, sinusoidal magnitude, and sinusoidal angle magnetization. The parameters of the motor taken are

$$
\begin{aligned}
& N_{p}=6, \quad \mathrm{~N}_{\mathrm{s}}=9, \quad b_{o}=1.5 \mathrm{~mm}, \quad R_{s}=16.95 \mathrm{~mm}, \\
& R_{r}=13.25 \mathrm{~mm}, \quad R_{m}=16.25 \mathrm{~mm}, \\
& B_{r}=1.2 \mathrm{~T}, \quad \mu_{1}=1000, \\
& \mu_{2}=1.05, \quad \mu_{3}=1000, \quad \alpha_{m}=0.8 .
\end{aligned}
$$

The results obtained by our model and the FEM are shown in Fig. 7 for radial magnetization, Fig. 8 for parallel magnetization, Fig. 9 for sinusoidal angle magnetization, and Fig. 10 for sinusoidal amplitude magnetization. In the analytical model 20 harmonics were considered, i.e., $\mathrm{n}=1,3, \ldots 21$ and $\mathrm{m}=$ $1,2, \ldots, 20$.

From the above figures it can be seen that results of analytical are close to the results of FEM. The radial component of the field obtained by the analytical method follows the FEM results in amplitude and waveform shape. However, at the edges of the slots the field due to FEM is higher than that obtained by analytical method. This difference can be attributed to the fact that at tooth edges flux concentration occurs and the relative permeance function is unable to take into account this flux concentration effect.

\section{BACK-EMF CALCULATION}

The voltage induced in the in the stator windings by varying magnetic field in the air gap is known as back-EMF. Since all coils in the stator can be described in terms of a sequence of equivalent single-tooth coils [12], hence the flux linked by each coil is the sum of fluxes linked by each individual tooth coils. 


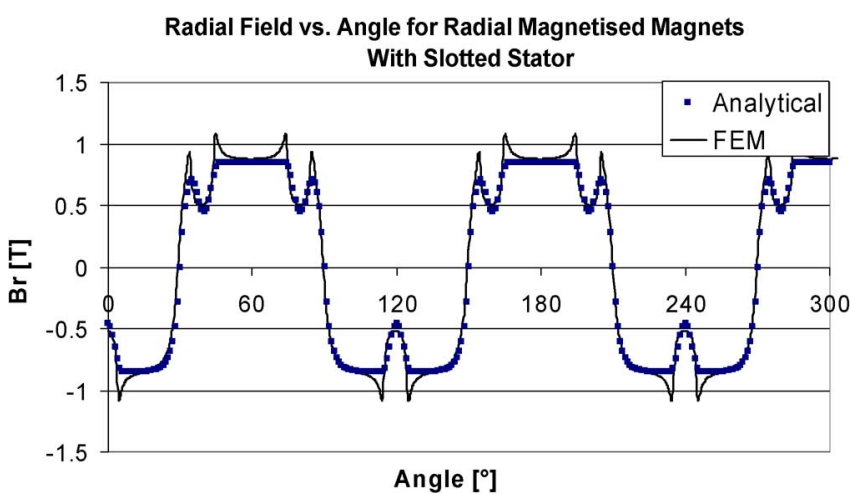

(a)

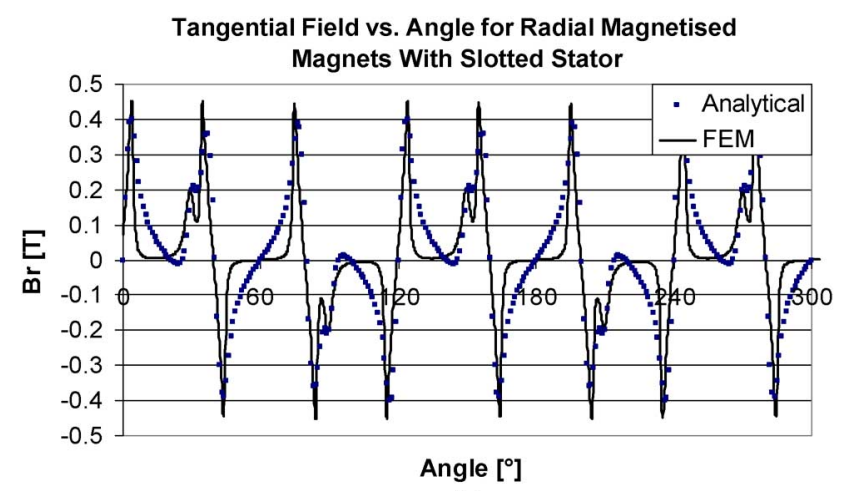

(b)

Fig. 7. Distribution of (a) radial component and (b) tangential component of air-gap field in a slotted motor with radially magnetized magnets $(\mathrm{r}=16.75 \mathrm{~mm})$

Fig. 11 shows a coil and its single-tooth equivalent. The flux linked by the coil in Fig. 11 is given by

$$
\phi_{c}(\theta)=\phi_{1}(\theta)+\phi_{1}\left(\theta-\theta_{s}\right)+\phi_{1}\left(\theta-\theta_{s}\right)
$$

where

$$
\begin{array}{ll}
\theta_{s} & \text { angular tooth offset; } \\
\phi_{1} & \text { flux linked by first tooth. }
\end{array}
$$

The flux linked by a tooth is given by

$$
\phi=\int \vec{B} \cdot d \vec{A} .
$$

In the above equation, by substituting $\vec{B}$ the radial component of the air-gap field at the stator inner surface (20) becomes

$$
\phi=\int_{-L / 2}^{L / 2} \int_{-\theta_{s} / 2}^{\theta_{s} / 2} B_{r I}(r, \theta) R_{s} d \theta d z
$$

where $B_{r I}(r, \theta)$ is the air-gap field at the stator surface, $R_{s}$ is the inner radius of the stator (Fig. 1), and $L$ is the length of the motor. In the above equation, the integrand is independent of the axial direction. Hence, the above equation can be simplified as

$$
\phi=\frac{2 L R_{s}}{N_{p}} \int_{-\theta_{s} / 2}^{\theta_{s} / 2} B_{r I}(r, \theta) d \theta .
$$

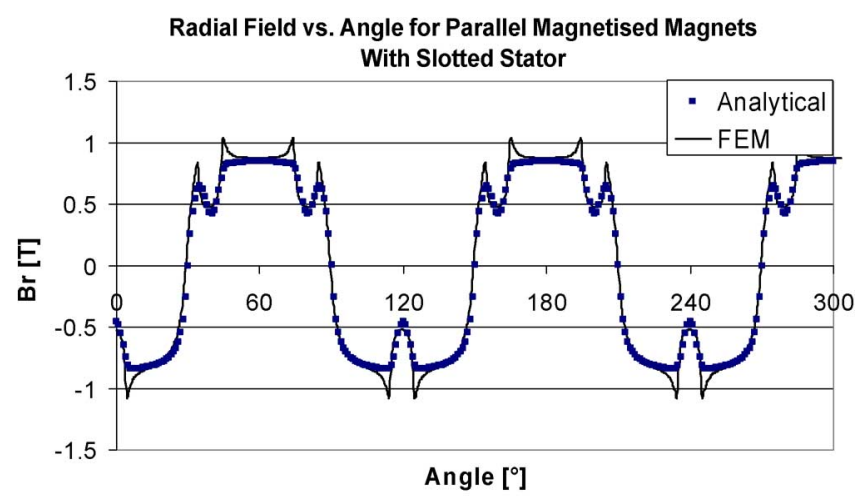

(a)

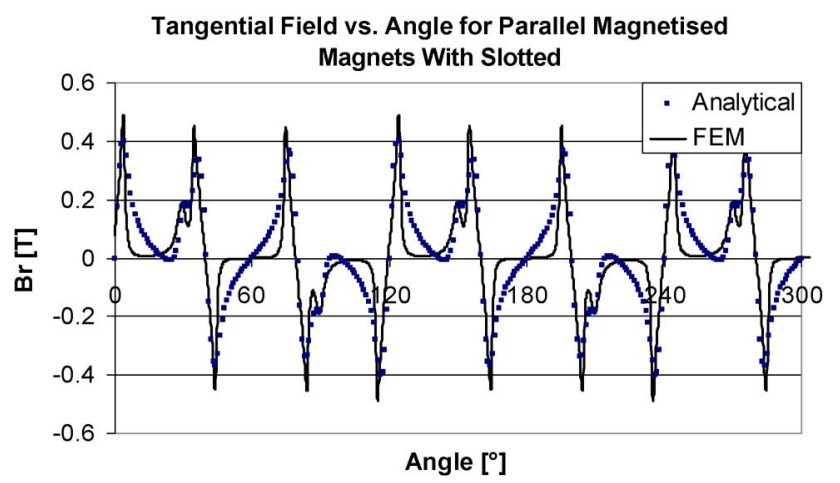

(b)

Fig. 8. Distribution of (a) radial component and (b) tangential component of air-gap field in a slotted motor with parallel magnetized magnets $(\mathrm{r}=16.75 \mathrm{~mm})$.

The back-EMF of a single-tooth equivalent coil is given by

$$
e=N_{\text {turns }} N_{p} / 2 \omega \frac{d \phi}{d \theta}
$$

where $N_{\text {turns }}$ is the number of turns in the coil.

The back-EMF of a general coil is the sum of back-EMFs of its single-tooth equivalent coils. For the coil shown in Fig. 11, the back-EMF is given by

$$
e_{c}(\theta)=e_{1}(\theta)+e_{1}\left(\theta-\theta_{s}\right)+e_{1}\left(\theta-\theta_{s}\right)
$$

Having developed the necessary set of equations, this model is tested and results for the air-gap field are compared with FEM. The air-gap field density is not compared with the experimental result due to difficulty in measurement of the air-gap field. However, since the back-EMF is evaluated from the air-gap field distribution, comparison of experimental values of back-EMF and those obtained by the analytical methods implicitly establish the validity of the analytical methods.

The results obtained by analytical method for back-EMF (back-EMF is expressed as voltage constant $k_{e}$ ) are compared by the experimental values of the back-EMF. The parameters of the motor are as follows:

$$
\begin{aligned}
& N_{p}=8, \quad \mathrm{~N}_{\mathrm{s}}=12, \quad R_{s}=11 \mathrm{~mm}, \quad R_{r}=9.5 \mathrm{~mm}, \\
& \mathrm{R}_{\mathrm{o}}=19.5, \quad R_{m}=10.8, \quad B_{r}=0.573 \mathrm{~T}, \quad \mu_{1}=550, \\
& \mu_{2}=1.05, \quad \mu_{3}=550, \quad \alpha_{m}=1, \quad N_{\text {turns }}=10 .
\end{aligned}
$$




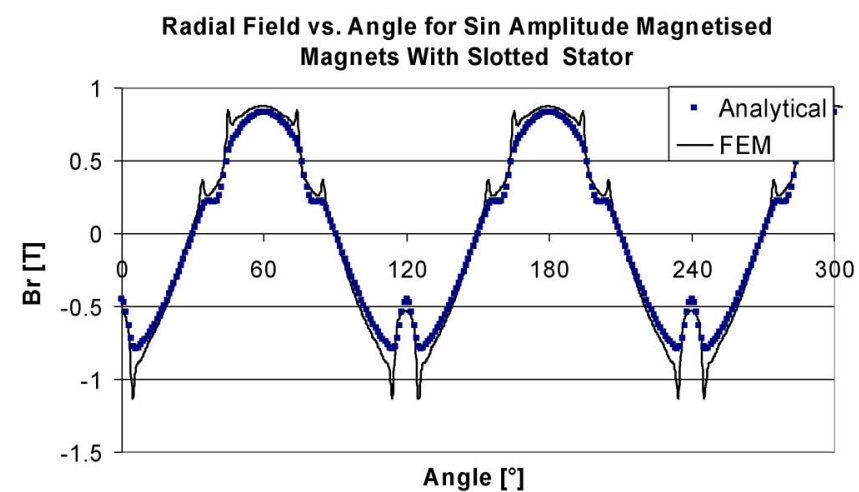

(a)

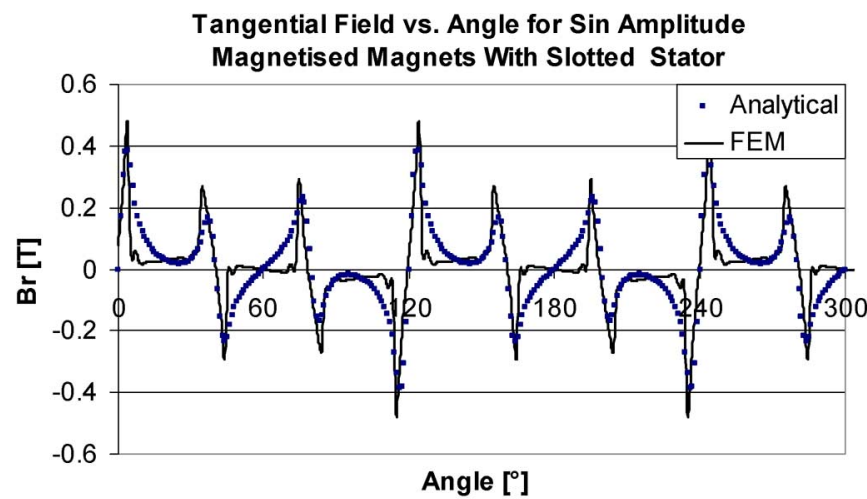

(b)

Fig. 9. Distribution of (a) radial component and (b) tangential component of air-gap field in a slotted motor with sin amplitude magnetized magnets $(\mathrm{r}=$ $16.75 \mathrm{~mm}$ ).

The four coils of a phase are connected in parallel and the phases are connected in series. The rotor and stator is yoke is made of M-250-35A steel. The $B-H$ curve of this steel is shown in Fig. 12

From Fig. 12, it can be seen that the material has linear characteristics upto $1.2 \mathrm{~T}$, i.e., the relative permeability of the material is constant it is $\mu_{1}=550$. This value of relative permeability is used in the analytical model. The back-EMF obtained by the analytical method for the above motor and the experimental values are shown in Fig. 13. From the above comparison, it can be seen that the results obtained by analytical method for $k_{e}$ closely resemble those of the experimental results.

\section{Cogging Torque Calculation Using MaXwell STRESS TENSOR}

The Maxwell stress gives the force per unit area produced by the magnetic field on a surface. In differential form, it is described as

$$
d f=\frac{1}{2}(\vec{H}(\vec{B} \bullet \hat{n})+\vec{B}(\vec{H} \bullet \hat{n})-(\vec{H} \bullet \vec{B}) \hat{n})
$$

where $\hat{n}$ denotes the direction normal to the surface at the point of interest. The net force on an object is obtained by creating a surface totally enclosing the object of interest and integrating the magnetic stress over that surface. In rotating motors, the tangential component of the force contributes to the torque. The

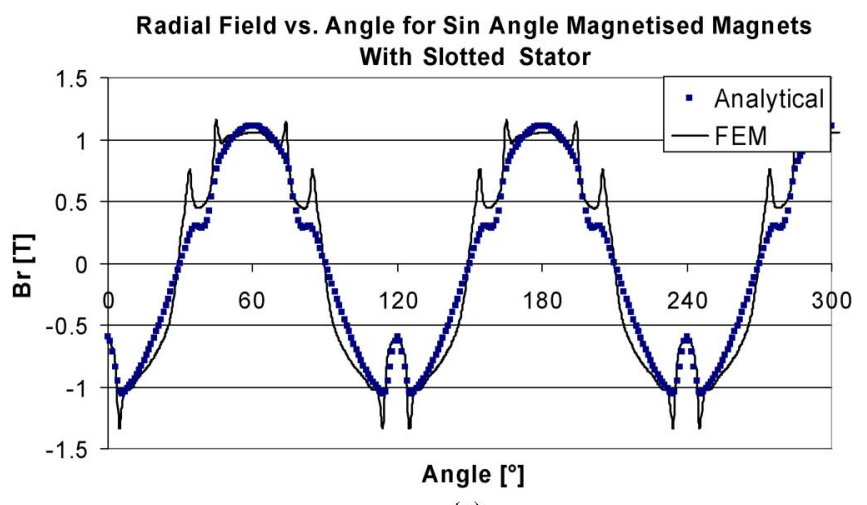

(a)

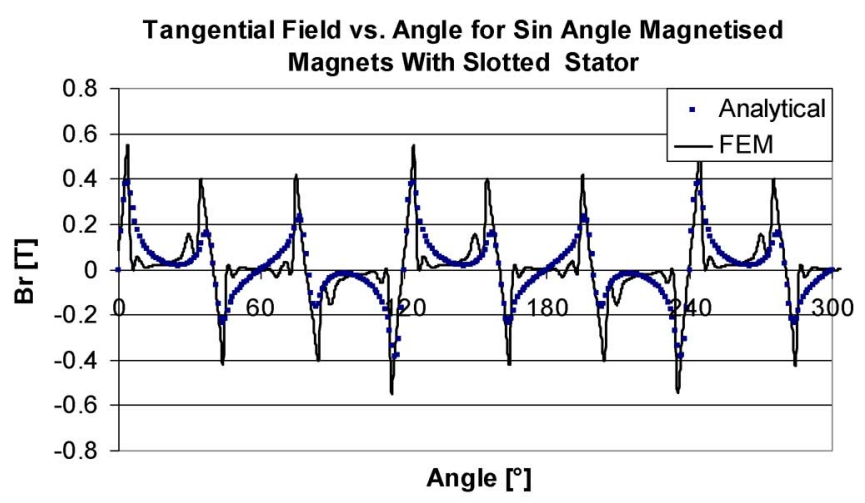

(b)

Fig. 10. Distribution of (a) radial component and (b) tangential component of air-gap field in a slotted motor with sin angle magnetized magnets $(\mathrm{r}=$ $16.75 \mathrm{~mm}$ )

tangential component of the force and torque acting on the surface that encloses the rotating part of the motor is given by

$$
f=\frac{1}{\mu_{0}} \int_{S} B_{r} B_{\theta} d S
$$

and

$$
T=r f .
$$

Using (44), the cogging torque in the motor can be determined. The comparison of the cogging torque obtained by the analytical model and the FEM are shown below. The parameters of the motor, for radial and parallel magnetized magnets, for which the cogging torque was calculated, are

$$
\begin{aligned}
& N_{p}=6, \quad \mathrm{~N}_{\mathrm{s}}=9, \quad b_{o}=1.5 \mathrm{~mm}, \quad R_{s}=16.95 \mathrm{~mm}, \\
& R_{r}=13.25 \mathrm{~mm}, \quad R_{m}=16.25 \mathrm{~mm}, \quad B_{r}=1.2 \mathrm{~T}, \\
& \mu_{1}=1000, \quad \mu_{2}=1.05, \quad \mu_{3}=1000, \quad \alpha_{m}=0.8 .
\end{aligned}
$$

From Fig. 14(a), it can be seen that the result of the cogging torque obtained by the analytical model is close to the FEM result. The shape of cogging torque from FEM is similar to that of the analytical values. For parallel magnetized magnets, the results of cogging torque obtained by analytical method and FEM are shown in Fig. 14(b). In this case, also, the shape of the cogging torque from analytical model and FEM is similar and the values are almost the same. The maximum value of cogging 


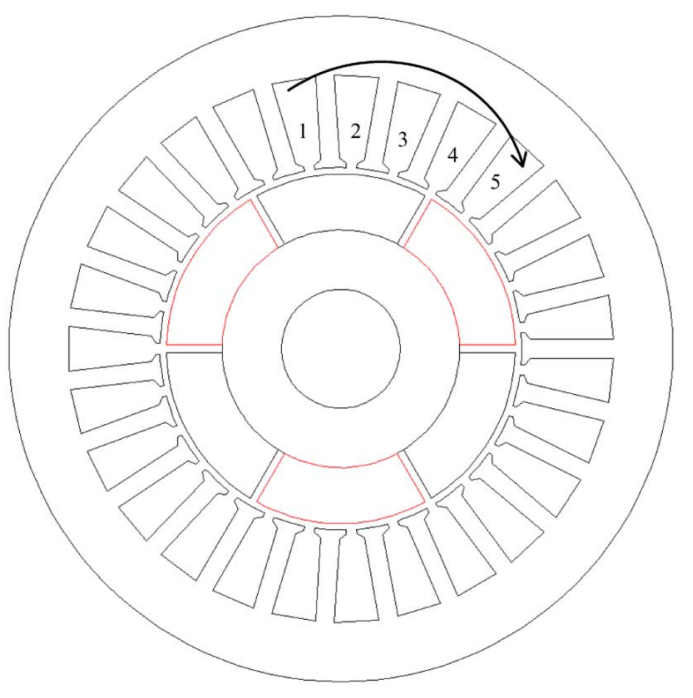

(a)

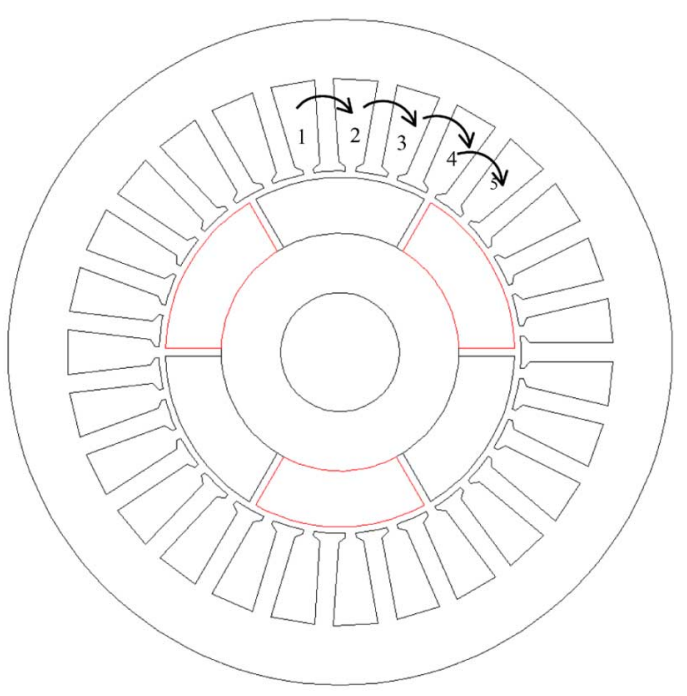

(b)

Fig. 11 (a) Coil with a span of 4 slot pitches. (b) Single-slot equivalent.

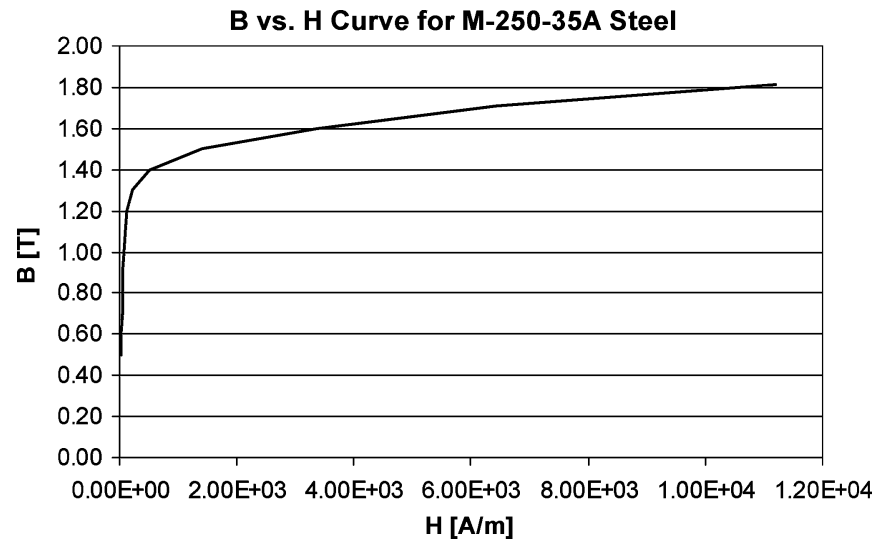

Fig. 12. $B-H$ curve of M-250-35 A steel.

torque calculated by analytical model, for both radial and parallel magnetized magnets, is about $7 \%$ higher than the values obtained by FEM.

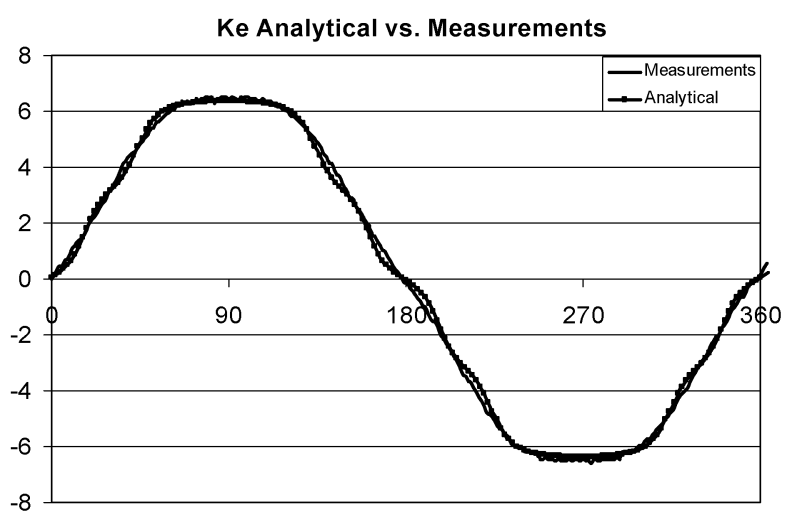

Fig. 13. Analytical result of back-EMF.

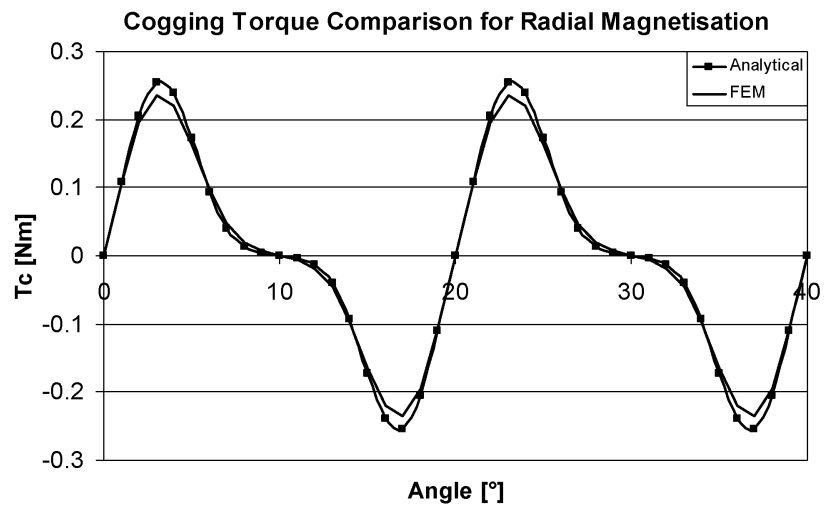

(a)

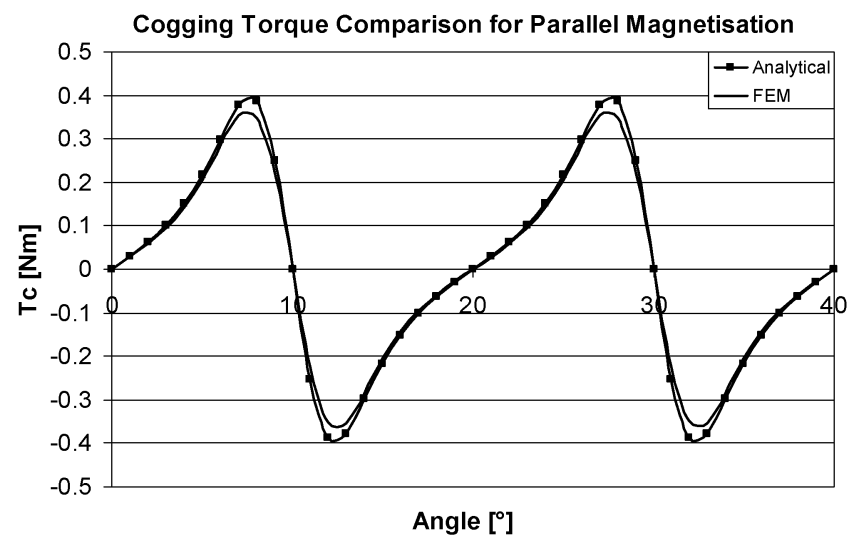

(b)

Fig. 14. Comparison of cogging torque obtained by analytical model and FEM for (a) radially magnetized magnets and (b) parallel magnetized magnets.

\section{CONCLUSION}

An analytical technique, to predict the air-gap field distribution due to permanent magnets mounted on the rotor of a BLDC motor with slotless and slotted stator, has been presented in this paper. This model takes into account that the stator yoke has finite permeability and finite thickness. Different types of magnetization of the permanent magnets viz., radial, parallel, sinusoidal magnitude, and sinusoidal angle magnetization have been considered. The model developed in this work has been validated by finite-element analysis. There is discrepancy between the radial component of the air-gap field obtained by analytical method and FEM. This difference is due to flux concentration 
effect at the tooth edges and the relative permeance function is unable to take into account this effect. Furthermore, the analytical cogging torque results obtained by analytical model and FEM were compared and were found to be similar to each other except that the maximum value of cogging torque by analytical method was about $7 \%$ higher than the values of FEM results. The analytical model was also compared with experimental results based on back-EMF values and both the results were in good agreement. The major advantage of the analytical method is that it is very fast compared to FEM and can be used in the initial multiobjective optimization of the BLDC motor.

\section{ACKNOWLEDGMENT}

The authors wish to thank Delft University of Technology, The Netherlands, for the kind support.

\section{REFERENCES}

[1] B. Heller and V. Hamata, Harmonic Field Effects in Induction Machines. New York: Elsevier, 1977.

[2] J. D. L. Ree and N. Boules, "Magnetic shaping to reduce induced voltage harmonics in PM machines with surace mounted magnets," IEEE Trans. Energy Convers., vol. 6, no. 1, pp. 155-161, Mar. 1991.

[3] P. D. Evans and D. Brown, "Simulation of brushless DC drives," Proc. Inst. Elec. Eng., vol. B-137, pp. 299-308, 1990.

[4] J. D. L. Ree and N. Boules, "Torque production in permanent magnet synchronous motors," in IEEE Industrial Applications Annual Meeting Record, 1987, vol. 1, pp. 15-20.
[5] G. Eid and A. Mouillet, "Transistorised DC brushless micromotor with rare-earth permanent magnets," presented at the ICEM 84, Luassane, Switzerland, 1984.

[6] Q. Gu and H. Gao, "Effect of slotting in PM electrical machines," Elect. Mach. Power Syst., vol. 10, pp. 273-284, 1985.

[7] N. Boules, "Two dimensional field analysis of cylindrical machines with permanent magnet excitation," IEEE Trans. Ind. Appl., vol. IA-20, no. 5, pp. 1267-1277, Sep. 1984

[8] N. Boules, "Prediction of no-load flux density distribution in PM machines," IEEE Trans. Ind. Appl., vol. IA-21, no. 3, pp. 633-643, May 1985.

[9] Z. Q. Zhu, D. Howe, E. Bolte, and B. Ackermann, "Instantaneous magnetic field distribution in brushless permanent magnet DC motors part I: Open-circuit field," IEEE Trans. Magn., vol. 29, no. 1, pp. 124-135, Jan 1993.

[10] K. F. Rasmussen, "Analytical prediction of magnetic field from surface mounted permanent magnet motors," presented at the Int. Conf. Electrical Machines and Drives, Seattle, WA, 1999.

[11] Z. Q. Zhu and D. Howe, "Improved analytical model of predicting the magnetic field distribution in brushless permanent magnet," IEEE Trans. Magn., vol. 38, no. 1, pp. 229-238, Jan. 2002.

[12] D. C. Hanselmann, Brushless Permanent Magnet Motor Design. New York: McGraw Hill, 2004

[13] Z. Q. Zhu, D. Howe, E. Bolte, and B. Ackermann, "Instantaneous magnetic field distribution in brushless permanent magnet DC motors: Part III: Effect of stator slotting," IEEE Trans. Magn., vol. 29, no. 1, pp. 152-157, Jan. 1993.

[14] U. Kim and K. Lieu, "Magnetic field calculation in permanent magnet motors with rotor eccentricity: With slotting effect considered," IEEE Trans. Magn., vol. 34, no. 4, pp. 2253-2266, Jul. 1998.

Manuscript received September 25, 2006; revised June 12, 2008. Current version published September 19, 2008. Corresponding author: P. Bauer (e-mail: P.Bauer@TUDelft.nl). 